\title{
Anabases
}

ANABASES Traditions et réceptions de l'Antiquité

$20 \mid 2014$

Varia

\section{Traduire en hexamètres français : une contradiction dans les termes?}

Juliette Lormier

\section{(2) OpenEdition}

1 Journals

Édition électronique

URL : http://journals.openedition.org/anabases/5014

DOI : 10.4000/anabases. 5014

ISSN : 2256-9421

Éditeur

E.R.A.S.M.E.

Édition imprimée

Date de publication : 1 novembre 2014

Pagination : 174-191

ISSN : 1774-4296

\section{Référence électronique}

Juliette Lormier, «Traduire en hexamètres français : une contradiction dans les termes ? ", Anabases

[En ligne], 20 | 2014, mis en ligne le 01 novembre 2017, consulté le 20 octobre 2019. URL : http:// journals.openedition.org/anabases/5014 ; DOI : 10.4000/anabases.5014

(c) Anabases 


\section{Traduire en hexamètres français : une contradiction dans les termes?}

JULIETTE LORMIER

Homère ! à ce grand nom, du Pinde à l'Hellespont, Les airs, les cieux, les flots, la terre, tout répond. Monument d'un autre âge et d'une autre nature, Homme! l'homme n'a plus le mot qui te mesure!

Lamartine $^{1}$

Malgré le silence des Anthologies de poésie française sur ce fait singulier dans l'histoire littéraire, ainsi que le peu d'intérêt que lui accorde encore aujourd'hui la recherche universitaire $^{2}$, nombreux sont les poètes français qui ont tenté, $\mathrm{du} \mathrm{XVI}{ }^{\mathrm{e}}$ siècle à nos jours, de mesurer leurs vers sur ceux des anciens, c'est-à-dire, dans un processus que l'on pourrait presque qualifier de translatio studii métrique, d'adapter au français les principes de la prosodie grecque et/ou latine ancienne(s). Séduits par l'idée de se mesurer à eux, ces auteurs ont pour la plupart inventé, théorisé ${ }^{3}$ et mis en pratique un système

1 A. De Lamartine, Euvres complètes. Tome 1, Paris, 1850, p. 427-428.

2 L'ouvrage qui fait clairement exception à ce constat général est celui de G. Peureux intitulé La fabrique du vers, paru aux éditions du Seuil à Paris, en 2009, dans la collection "Poétique "; un peu moins récemment, celui d'E. Etkind, Un art en crise. Pour une poétique de la traduction poétique, Paris, L'Âge d'Homme, 1982.

3 L'existence de deux traités composés par Baïf, l'un « d'art métrique », l'autre " de prononciation » du français, que nous aurions perdus, mentionnés par La Croix du Maine dans sa Bibliothèque éditée en 1584, est extrêmement discutable, quoique M. Augé-CHIQueT semblât y souscrire, cf. La Vie, les idées et l'oeuvre de Jean-Antoine de Baïf, Paris-Toulouse, 1909 , p. 347. 
permettant de former des pieds en français. Des pieds ? Qu'est-ce à dire ? Précisément ici, selon la définition purement classique de la métrique ancienne, divers groupements repérables de syllabes longues et de syllabes brèves qui, une fois disposés ensemble dans un nombre et un ordre particulier, forment un vers grec ou latin ${ }^{4}$.

\section{Plusieurs poésies françaises " à l'antique" ", plusieurs hexamètres}

Il faut dès maintenant préciser notre propos et distinguer divers types d'expériences métriques "à l'antique " en France. Si les auteurs français apprennent à percevoir comme à apprécier des alternances de durée constituant une succession de pieds dans les vers grecs et latins (succession marquée elle-même par le retour régulier d'un ictus rythmique ${ }^{6}$ ), le chemin est encore long de cette métrique quantitative à son adaptation dans leur langue. Cela tient d'une part à la primauté accordée très tôt à la métrique syllabique en France et à l'idée qu'il n'existe pas de prosodie du français ${ }^{7}:$ la possibilité d'une métrique quantitative a été ainsi promptement évacuée, notamment grâce à une catégorie interprétative plutôt critiquable que l'on a pu nommer le " génie de la langue française ". Ainsi, à mesure que l'histoire littéraire s'est écrite, les obstacles idéologiques ont freiné les tentatives, puis grandi, enfin gagné en légitimité apparente par le résultat même de leur effet dissuasif sur les poètes. D'autre part, pour n'importe quel auteur, l'adaptation passe d'abord par une perception toute personnelle du vers antique et de son rythme. Cette perception a son importance dans le travail postérieur de restitution $^{8}$ : ainsi, Baïf dans sa traduction des Besognes et des Jours ne recrée pas ce qui serait objectivement l'" hexamètre historique " d'Hésiode, tel qu'il aurait été transmis par le

4 Ces pieds sont des constituants de ce que les linguistes appellent la métrique morique, c'est-à-dire fondée sur la durée (puisqu'une «more », terme issu du grec, signifie une unité de durée).

5 Nous utilisons pour le moment l'expression "à l'antique » faute de mieux, pour englober autant les véritables calques des patrons métriques anciens que les transpositions uniquement inspirées de ceux-ci et qui peuvent reposer sur un autre élément d’alternance que la quantité.

6 L'ictus est le battement du temps fort de la mesure, marqué soit en tapant du pied, soit en frappant dans ses mains, pour les anciens : en latin, ictus signifie " choc, coup ", puis plus spécifiquement "battement de la mesure ». Son existence est encore débattue, de même que son rôle dans l'enseignement des métriques anciennes en France, notamment à la Renaissance.

7 C'est-à-dire aucune opposition de durée flagrante entre les diverses voyelles et syllabes du français, propice à l'élaboration d'une poésie quantitative.

8 La connaissance préalable de la métrique ancienne influe également sur la restitution, mais pas toujours décisivement. Voir la comparaison des hexamètres de BAÏF et BRUNET plus bas. 
texte dans sa forme originelle et idéale, mais plutôt ce qu'il perçoit de cet hexamètre, ce qui, rythmiquement lui semble caractéristique de ce vers. Bien sûr, la perception s'attache globalement aux mêmes qualités saillantes du vers pour la plupart des auteurs, mais les moyens mobilisés par eux pour recréer ce mètre tel que perçu peuvent être différents. Les poètes ont recours à divers éléments de saillance (syllabes allongées, accentuées, etc.) pour rendre précisément ce qui a été perçu (avec toute l'ambiguïté que cette dernière expression implique également : perçu en lecture intérieure, en récitation à mi-voix, en déclamation, en psalmodiant et en battant la mesure ?).

D'un point de vue historique, les expériences successives de composition de poésie à l'antique (nommée également poésie " métrifiée ", " mesurée ", "métrique " ou encore "prosodique ") ne se sont jamais inscrites dans un courant littéraire majeur ou dominant qui eût pu donner à ceux qui s'y risquaient l'élan fédérateur propice à poursuivre longuement l'entreprise, et par conséquent, à lui donner un achèvement certain. Bien au contraire, nous traitons ici d'expériences et de systèmes parallèles, et plus précisément marginaux. De fait, en matière de poésie à l'antique, les tentatives sont nombreuses, puisqu'elles parsèment distinctement l'histoire littéraire française, mais elles restent pour la plupart (le XVI $I^{\mathrm{e}}$ siècle et probablement le $\mathrm{XXI}^{\mathrm{e}}$ exceptés ${ }^{9}$ ), le fait de groupuscules d'auteurs s'attelant à la tâche très ponctuellement, quand il ne s'agit pas de l'expérience unique et inouïe de quelque poète isolé, faisant figure de véritable aérolithe dans son quart de siècle, et qui, pour cette raison précisément, est rapidement découragé ${ }^{10}$. Malgré cette représentation restreinte dans la chronologie littéraire française, si l'on dresse un panorama historique de ce type d'expériences, deux remarques s'imposent : en premier lieu, un grand nombre de tentatives se situe dans le domaine de la traduction, qu'elle soit de textes anciens (Hésiode, Homère, Pindare, Sappho, Catulle) ou modernes (Hölderlin). Dans ce cas, les questions de composition de l'hexamètre ou d'autres vers anciens s'articulent aux divers problèmes posés par la traduction dite " poétique ${ }^{11}$ ». En outre, les techniques de restitution peuvent changer en fonction

9 Nous exceptons la vogue des vers mesurés composés pour être mis en musique dès le milieu du XVI ${ }^{\mathrm{e}}$ siècle et qui enthousiasma un grand nombre de poètes jusqu'aux années 1570, notamment dans l'entourage de BAÏF ou parmi ses disciples (tel Nicolas RapiN); actuellement, la compagnie Démodocos, réunie autour de Philippe BRUnET, mène ses propres expériences en traduction rythmée à l'antique, tout comme d'autres traducteurs d'Homère en hexamètres, dont une partie des travaux sont réunis dans ce volume.

10 Quoique rapidement dissuadé par Francis Jammes, nous pensons ici à Paul-Jean ToulET qui publia dans sa jeunesse une série d'élégies et d'épigrammes à la manière de BAÏF intitulées "Entr'actes ", en 1898, dans la Revue blanche, mais nous pouvons citer aussi plus tôt, en Belgique, le poète francophone André VAN Hasselt qui publia en 1862 Poèmes, paraboles, odes et études rythmiques dont Verlaine fait mention avec enthousiasme dans une lettre à Émile Blémont, le 25 juin 1873, voir P. Verlaine, Euvres poétiques complètes, Gallimard, Pléiade, Paris 1968, p. 1149-1150.

11 Voir E. ETKIND, Un art en crise, p. XI : pour Etkind, la traduction poétique correspond à la traduction versifiée d'un poème versifié - cette définition est bien entendu discutable. 
de l'émergence progressive de traités théoriques propres à influencer ces expérimentateurs. Il n'existe donc pas une seule poésie "à l'antique » française, mais plusieurs, pas un seul hexamètre français, mais plusieurs. Pour aller plus loin encore, nous allons voir dans cette étude qu'il n'existe pas un hexamètre français de traduction (qui serait adopté de façon univoque dans une espèce de rapport «naturel » à la langue) mais plusieurs, selon les principes de restitution appliqués par tel ou tel auteur et, encore en amont, selon sa perception personnelle de l'hexamètre ancien.

\section{La traduction poétique : problématiques et réponses hexamétriques}

Vouloir traduire le texte homérique ou hésiodique en hexamètres révèle en soi une prise de position poétique ${ }^{12}$. D'une part, le refus de la prose que cela implique n'a rien d'anodin lorsque l'on sait que la traduction en prose de l'épopée connut dès le $\mathrm{XVII}^{\mathrm{e}}$ siècle une popularité écrasante en France : systématiquement située du côté du vrai sens et de la transmission de l'idée juste, on lui opposait en effet la traduction en vers, trop rigide, aux dires de ses détracteurs, et laissant trop peu de libertés quant au choix et à l'agencement des mots. Depuis quelques années cependant, plusieurs traductions en vers saluées par la critique et les lecteurs tendent à inverser cette tendance et à redonner leur juste place aux divers mouvements rythmiques et sonores du poème originel, et parmi elles notamment l'Odyssée de Philippe Jaccottet ${ }^{13}$. La quête du sens ne semble plus dans les esprits remise en cause par le recours aux vers. D'autre part, dans ce choix délibéré de l'hexamètre, une attention toute particulière est portée au rythme originel du poème ancien, soit que le traducteur croie fermement en sa capacité à transmettre plus adéquatement la parole épique (mais ne devrions-nous pas parler ici de chant ?), soit qu'il ait comme souci profond de rendre le poème dans toute son essence, dans cette nature unique, à la fois plastique et sémantique de la parole scandée, ne cessant jamais de concevoir ce chant révéré comme un tout. Le choix d'une traduction en hexamètres peut bien entendu naître de ces deux élans à la fois, mais il peut aussi être guidé, comme dans le cas de Baif, par le souci de défendre et illustrer sa langue

12 Nous entendons ici le terme de poétique en tant que «facture, fabrication de l'œuvre poétique ".

13 L'Odyssée, traduction, notes et postface de Pн. JАсCотTEт, Paris, Club français du Livre,

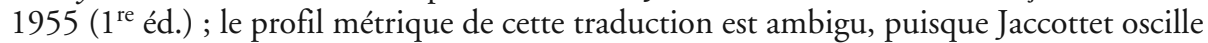
entre les vers de quatorze syllabes, majoritaires, les alexandrins "utilisé[s] de préférence pour les vers formulaires introduisant un interlocuteur ", et parfois, les décasyllabes. Le poète explicite sa position dans la partie " Note sur la traduction » (p. 411, éd. 2004, Paris, La Découverte). 
en la mesurant à celle des anciens et en lui accordant, dans un processus d'aemulatio déjà cher aux Romains, une noblesse antique ${ }^{14}$.

Mais choisir de traduire en hexamètres est aussi révélateur d'une poétique de la traduction telle qu'elle a pu être théorisée en France dès les années 1980-1990 par Efim Etkind ou Henri Meschonnic ${ }^{15}$. Selon Etkind, le poème scelle l'union d'un sens et d'une forme et ne doit par conséquent jamais être traduit partiellement. L'auteur va plus loin : il ne faut d'après lui jamais tenter de traduire le sens porté par un poème sans essayer simultanément d'en traduire le rythme, la matière sonore et la composition ${ }^{16}$. Meschonnic quant à lui, tout en se gardant de confondre vers et poésie, relativise la crise de la traduction poétique qu'avait postulée Etkind en France et résout dans sa définition du rythme le traditionnel conflit qui opposait jusqu'alors forme et fond, forme et sens, au cœur du signe :

" [...] je prends le rythme comme l'organisation et la démarche même du sens dans le discours. C'est-à-dire l'organisation (de la prosodie à l'intonation) de la subjectivité et de la spécificité d'un discours : son historicité. Non plus un opposé du sens, mais la signifiance généralisée d'un discours. Ce qui s'impose immédiatement comme l'objectif de la traduction. L'objectif de la traduction n'est plus le sens, mais bien plus que le sens, et qui l'inclut : le mode de signifier ${ }^{17}$."

Affirmant ainsi à son tour l'union indissoluble de la forme et du sens dans le poème, Meschonnic définit la grande traduction comme " une contradiction tenue, au sens contraire de la conception courante qui tend à la résoudre dans une direction ou une autre, entre les deux termes de la dualité du signe : vers la langue de départ ou vers la langue d'arrivée, vers la forme ou vers le sens, vers l'exactitude ou vers une beauté d'adaptation, toujours dans l'effacement du traducteur ${ }^{18}$ ». Le choix de

14 Voir le poème «Au Roi » dans les Étrénes de BAïF ; à ce sujet, on remarque qu’il ne faut pas éluder l'historicité de telles tentatives métriques en français : elles prennent généralement place à des périodes de fortes mutations dans l'histoire de la poésie française, notamment de fixation de règles de composition nouvelles, comme au XVI ${ }^{\mathrm{e}}$ siècle, ou de révolte contre les règles préétablies, comme à la fin du XIX siècle (Toulet compose tandis qu'émerge le vers libre).

15 Voir E. Ethind, Un art en crise, et H. Meschonnic, Poétique du traduire, Lagrasse, Verdier, 1999.

16 ETKIND, Un art en crise, p. XI : «La poésie, c'est l'union du sens et des sons, des images et de la composition, du fond et de la forme. Si, en faisant passer le poème dans une autre langue, on ne conserve que le sens des mots et les images, si on laisse de côté les sons et la composition, il ne restera rien de ce poème. Absolument rien. »

17 Meschonnic, Poétique, p. 99-100, nous soulignons.

18 Meschonnic, Poétique, p. 55 ; Brunet ne s'oppose pas, pour sa part, à l'effacement du traducteur, dans sa préface à l'Iliade, au contraire, il remet en cause la légitimité d'un style personnel du traducteur dans son ouvre pour comparer son effacement à ce que l'on pourrait appeler la « discrétion auctoriale » d'Homère : «Le renoncement à toute initiative 
l'hexamètre comme vers de traduction de l'épopée ancienne en français pourrait se situer dans cette « contradiction tenue » définie par l'auteur : de fait, par sa longueur, à la fois considérable et variable (par opposition aux formes syllabiques fixes tel que l'alexandrin ou le décasyllabe : dans le cas de la transposition presque "classique " de Baif, l'hexamètre compte de treize à dix-sept syllabes, dans le cas de la restitution de Brunet, en moyenne entre treize et seize), l'hexamètre est un vers doté d'une souplesse certaine qui ne contraint pas excessivement le traducteur soucieux simultanément du sens. Le rythme de ce mètre, une fois apprivoisé pour la composition, senti comme un vecteur et un allié du sens, peut même lui devenir assez aisément consubstantiel. Ainsi, l'hexamètre pourrait bien représenter un candidat idéal pour l'avènement d'une phono-sémantique, ou bien de ce que Meschonnic nomme lui-même " la sémantique prosodique et rythmique ${ }^{19}$ ", ce tout qui correspond au poème. En outre, commentant ce qu'il nomme l'" effet de signifiance " dans deux hexamètres d'Homère (Iliade VIII, 64 et 65), Meschonnic affirme qu'ils constituent également un bon exemple de «l'agir du langage ${ }^{20}$ ", selon le postulat, fondamental pour le critique, que le texte est avant tout action plutôt qu'unique information, et que la traduction doit rendre non pas ce que les mots disent, mais «ce qu'ils font ${ }^{21}$ ". Si l'on suit ce raisonnement, on s'éloigne alors nettement de la conception classique d'un hexamètre qui servirait uniquement la traduction en tant que forme métrique. Au contraire, l'hexamètre de traduction, selon cette théorie moderne, serait l'élément clef d'une poétique créatrice, visant à recréer l'agir du langage contenu dans le texte ancien. Non pas la manière de rendre la parole hésiodique ou homérique, mais cette parole elle-même véhiculée dans son entier et son essence (dans les effets qu'elle peut produire à la lecture aussi ${ }^{22}$ ); un mélange homogène de l'esprit avec sa forme, ou, si l'on veut, la phono-sémantique originelle du poème, transmise de nouveau, quoique différemment, puisque dans la mesure des moyens d'expression en présence - et cela dans un refus permanent adressé à l'hyperlittéralité. Or, pour retrouver et transmettre cette phono-sémantique dans sa langue sans procéder à un calque stérile (parce qu'uniquement formel), le traducteur devra faire œuvre de créateur, de second auteur, refuser une part de son effacement traditionnel.

d'auteur, n'est-ce pas la force paradoxale d'Homère, profondément vouée à recevoir la voix de la Muse, comme celle de la traduction littéraire ? ", L'Iliade, Seuil, Paris, 2010, «Préface », p. 30.

19 Meschonnic, Poétique, p. 24 et p. 109, concernant un passage d'Homère, L'Iliade VIII, 64-65.

20 Meschonnic, Poétique, voir toute la section p. 139-141.

21 Meschonnic, Poétique, p. 140.

22 La traduction en hexamètres permet une redynamisation de la " fonction " du poème, élément essentiel pour ETKIND (voir Un art en crise, "Préface pragmatique ", p. 1-13) et qui appelle une traduction sous peine de contresens absolu : dans le cas de l'hexamètre, cette fonction pourrait bien être le divertissement d'un auditoire par un récit oralisé et rythmé. 
Mais l'un des apports les plus importants de tels essais de traduction, nous semblet-il, est d'ordre idéologique : leur existence même bouscule les conceptions normatives de la langue, de la métrique, enfin de la poésie françaises. Le simple fait de s'interroger sur la «faisabilité » d'une telle métrique à partir du français, mais également les efforts et réussites des traducteurs dans ce domaine, battent en brèche les préjugés les plus anciens sur la nature de la langue française, pour le dire plus traditionnellement, sur son " génie ». Ce qui change, précisément, c'est l'idée que l'on se faisait des exigences de sa langue, c'est le champ des possibles que l'on imaginait beaucoup plus restreint, dans cette idée que la métrique serait uniquement et unilatéralement dépendante du langage, qu'elle en découlerait entièrement et nécessairement. Mais comme le précise G. Peureux au sujet de la métrique française : "La métrique syllabique, en dépit de sa très large domination quantitative dans le corpus de la poésie française, est une métrique possible et non pas nécessaire ou naturelle pour le français, elle relève de choix et de la fixation d'habitudes dont procède sa pérennité ${ }^{23}$. " Les tentatives de traduction en hexamètres français sont ainsi autant de signes d'une "vie " métrique et poétique en dehors de l'ordre commun préétabli : elles constituent de véritables laboratoires métriques, permettant non pas de contraindre la langue, mais plutôt d'apprendre à mieux la connaître en s'essayant à explorer toute l'étendue de ses virtualités poétiques.

\section{Deux hexamètres français : Jean-Antoine de Baïf marchant au pas d'Hésiode...}

Il nous a semblé pertinent de nous pencher sur deux traductions distantes de presque cinq siècles l'une de l'autre, mais témoignant d'une même ambition chez leurs auteurs : celle de rendre dans la langue française le mouvement rythmique caractéristique de l'hexamètre grec. Il s'agira ici, dans une perspective comparatiste, de distinguer les divergences de méthode employées par ces deux traducteurs et partant, de définir précisément la nature de l'hexamètre chez l'un et chez l'autre.

Les Étrénes de poézie fransoeze an vers mezurés constituent un recueil de dix-neuf poèmes publié en 1574 à Paris chez Denys du Val. Parmi eux, quatre sont des traductions de gnomiques grecs : Hésiode, Pythagore, Phocylide qui est en réalité Simonide, et Naumaque. Il faut bien entendu ajouter à ces pièces versifiées l'"Abc du langage français " accompagné de la "Brève raison des mètres de ce livre », tous deux composés en prose, et dont la place témoigne d'un profond souci pédagogique de la part de l'auteur. Parmi les mètres employés dans ce recueil, l'hexamètre s'avère un objet d'étude extrêmement intéressant, puisqu'il constitue dans les Étrénes le seul vers dont le nombre de syllabes soit aussi variable, ou si l'on préfere, le moins « isosyllabique " qui soit, c'està-dire le plus éloigné de ce que la poésie française, définie traditionnellement comme 
"syllabique ", propose. Sans vouloir ici trancher la question du bien-fondé du système prosodique de Baïf, vivement discuté par les chercheurs ${ }^{24}$, nous aimerions apporter quelques éléments d'analyse concernant sa traduction d'Hésiode en hexamètres. Cette traduction est un élément central du recueil, tant par sa place que par son étendue. Concernant le travail du traducteur plus précisément, on ignore de quelle(s) édition(s) $\mathrm{du}$ texte hésiodique Baif disposait : travaillait-il directement sur le grec ou bien passaitil, comme c'était souvent le cas à l'époque, par une traduction latine du grec ? On constate l'extrême fidélité avec laquelle Baïf traduit le lexique d'Hésiode, mais aussi sa syntaxe, formée par groupes de mots de même nombre de syllabes dans la mesure du possible. De temps à autre, Baïf imite même la scansion des attaques d'hexamètre, le nombre de syllabes dans la nomination des dieux avec leurs épithètes, parfois les hiatus présents dans le texte grec ${ }^{25}$ :

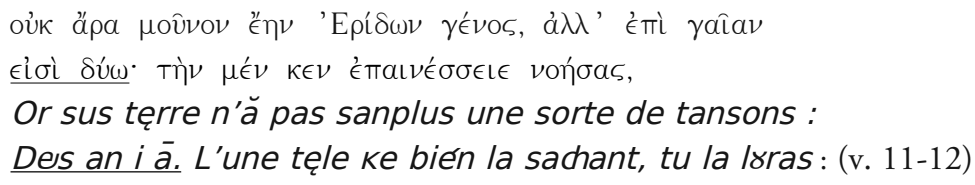

(Le vers s'ouvre sur un dactyle-calque : la succession des voyelles en hiatus, quoique le « $\mathrm{i}$ » puisse former une semi-consonne chez Baif par resyllabification avec le « $\mathrm{n} »$, semble imiter phoniquement l'ouverture hésiodique de l'hexamètre sur quatre syllabes dans les deux cas).

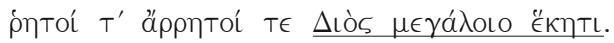

É renomés é non renomés, Du kran Jupitęr la volonte ! (v. 4)

24 Voir, pour les travaux récents et proposant d'importantes synthèses : Y.-CH. MoRIN, « La graphie de Jean-Antoine de Baïf : au service du mètre ", Nouvelle Revue du seizième siècle, 17 (1), 1999, p. 85-106, "L'hexamètre "héroïque " d'Antoine de Baïf », dans Métriques du Moyen Âge et de la Renaissance, D. Billy (dir.), Paris-Montréal, L'Harmattan, 1999, p. 163-184, enfin "La prononciation et la prosodie du français du XVI siècle selon le témoignage de Jean-Antoine de Baïf ", Langue française, $\mathrm{n}^{\circ}$ 126, 2000, p. 9-28, mais aussi O. BETTENS, "Une nouvelle voye pour aller en Parnasse" : modernité des vers mesurés à l'antique ", article à paraître en ligne dans le "Corpus EVE " (Émergence du Vernaculaire en Europe), université de Savoie [http://eve.revues.org] et "Octosyllabes, vers mesurés et effets de rythmes ", dans L'expérience du vers en France à la Renaissance, J.-C. MONFERRAN (dir.), Cahiers Saulnier, no 30, 2013, p. 185-213.

25 Dans l'édition des Étrénes, le caractère e symbolise la monophtongue [ø], et le caractère ૪, $[\mathrm{u}]$; e signale le plus souvent un son [e] fermé, et le ę un son $[\varepsilon]$, même si parfois Baïf interchange ces emplois; a représente le son [0]. Le caractère $\int$ marque la consonne $[s]$, $\varsigma$ la consonne [J], z la consonne [z]. Le circonflexe devait noter un allongement de la voyelle frappée avec une élévation de la voix retombant en fin d'émission (modulation sans doute comparable, pour Baif, à celle marquée par l'accent circonflexe grec). Dans ces vers, macrons et microns spécifient un poids syllabique qui aurait pu être ambigu pour le lecteur. 
(Baïf veille à employer le même nombre de syllabes pour terminer l'hexamètre : il a donc recours au nom romain de Zeus pour compenser la perte de syllabes sur l'adjectif « grand» traduisant le tétrasyllabe grec).

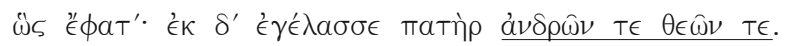

Einsi dit : e dez umeins é Dïes le Per' an Je mokant rit. (v. 59)

(Baïf semble travailler par syntagme en établissant des correspondances de nombre de syllabes (ici à une syllabe près avec la diérèse sur " Di-eux ») : il suit ainsi, autant que faire se peut, le rythme poétique du texte d'Hésiode, non sans parfois procéder à quelques inversions, pour placer en fin de vers un terme important à ses yeux et dont le sens est ainsi mis en valeur. Ce respect du nombre de syllabes ne fait pourtant pas de son système un système syllabique, puisqu'il n'y a pas de retour régulier d'un nombre de syllabes donné ; cependant, l'appréhension syllabique de certains syntagmes par le poète en grec comme en français reste manifeste).

Dans son hexamètre, le poète respecte autant que possible la synaphie du vers grec (les pieds ne coïncident pas avec un seul mot mais s'étendent sur plusieurs, dépassant les frontières de mots et les liant fortement les uns aux autres : oúva $\tau \varepsilon \varepsilon ı v$ signifiant

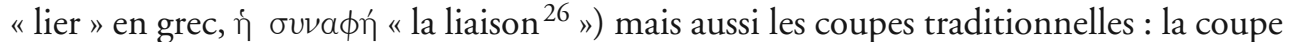
penthémimère après la cinquième position ou bien la coupe "trochaïque ", c'est-à-dire après la première brève de la sixième position, voire la coupe hephthémimère après la septième position métrique (qui s'offre plus fréquemment chez Baïf comme la meilleure des solutions, contrairement à ce que l'on observe en grec). En bon métricien antiquisant, Baiff applique en outre plusieurs lois anciennes comme celle de l'allongement par position. Mais le poète utilise également toutes les ressources de sa langue pour répondre au patron métrique qu'il a en tête : pour commencer son hexamètre sur une syllabe lourde, sur laquelle on insiste, il place à l'initiale des pronoms personnels, des conjonctions dotées d'une charge sémantique certaine, ou encore des substantifs monosyllabiques commençant par une voyelle et donc précédés de l'article défini élidé ; il emploie notamment des " e " féminins pour « faire » les brèves des dactyles, le plus souvent au cinquième, obligatoire :

$$
\begin{aligned}
& - \\
& \text { Lęs Sęzons chevelûs la paroęt de florętes du Printans : (v. 75) }
\end{aligned}
$$

Cette attention portée à la restitution de la prosodie ancienne s'accompagne d'un souci remarquable de fidélité au profil sonore du vers grec. La traduction de

26 Sur cette notion, voir J. IRIGOIN, « Césure et diction du vers. Quelques réalités linguistiques à ne pas oublier ", dans Autour de la césure. Actes du colloque Damon des 3-4 novembre 2000, M. Steinrück et A. Lukinovich (dir.), Berne, F. Spaltenstein, O. Bianchi (éds), 2004, p. 1-10.

27 La dernière syllabe de l'hexamètre est traditionnellement dite « indifférente », marqué par un $<X>$ dans le schéma métrique. 
Baiff témoigne en effet de sa perception phonique du poème par l'adaptation qu'elle propose en français des échos sonores et autres jeux d'assonances ménagés par Hésiode. Il reprend par une rime (très rare dans le poème) l'emploi de participes construits sur

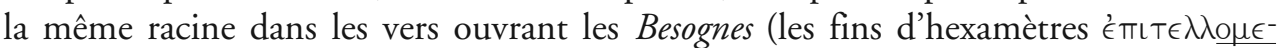
$\underline{v a ̉ \omega \nu}$ et $\delta v \sigma o \mu \in v a ́ \omega \nu$ deviennent resourdront, descendront, repérables dans un poème non rimé), mais il adapte également le jeu sonore extrêmement travaillé du v. $354 \mathrm{du}$ proème,

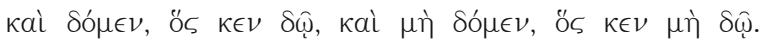

en traduisant :

Faut doner a donebién : ex ne rién doner, a ne donant rien.

Le jeu assonantique d'Hésiode, appuyé par le fait que chaque subordonnée relative propose, de façon quasi anagrammatique les mêmes sonorités que les principales, a bien été repéré par Baïf qui tente de le restituer à la fois par un travail de composition lexicale et par l'introduction d'assonances (donner / donne-bien / donnant rien). Baïf prête fortement attention au profil phonique du vers grec et sa perception de l'hexamètre d'Hésiode n'est pas uniquement graphique (il ne serait pas inconséquent de présumer que Baïf lisait ces hexamètres grecs à haute voix pour en élaborer la traduction).

Si l'on se familiarise avec sa graphie, mais aussi avec ses règles métriques et prosodiques, et si l'on mémorise le patron avec ses quelques substitutions, il est possible de lire les Besognes et les Jours de façon hexamétrique, c'est-à-dire proprement " mesurée à l'antique " (voire en frappant l'ictus !) ${ }^{28}$. S'agit-il ici d'un calque du modèle antique plutôt que d'un équivalent métrique ? Il semblerait bien. Ce qui ne veut pas dire que ce calque ne fonctionne pas, ne puisse pas être réalisé, puisque les catégories prosodiques de Baïf relèvent d'un système pour le moins strict et rigoureux ${ }^{29}$. Le poète s'astreint très clairement à respecter les longues et brèves attendues selon les positions métriques (l'expression graphique des hexamètres en témoigne, qui joue un rôle éminent de "guide " métrique), le patron abstrait du mètre exerce donc un rôle fort dans ce système, au point qu'on pourrait taxer l'auteur d'applicationnisme ou de rigidité. Cependant, indépendamment de la métrique, le jeu complexe des accents, des variations mélodiques, des pauses marquées ou non à tel ou tel endroit du vers, doivent contribuer à lui donner, à la diction, sa variété autant que son caractère inouï. Traduire Hésiode de cette façon, c'est donner au lecteur comme à l'auditeur matière à aiguiser sa perception, enfin matière à se familiariser avec l'inconnu, mais pas avec l'impossible.

28 Nous parlons ici de lecture intérieure ou bien à haute voix, même si cette dernière est conseillée, tout particulièrement dans le cas de l'hexamètre, vers typiquement épique, qui ne se pense pas, pour les anciens, sans une profération en public; nous ignorons si Baïf destinait les traductions des Étrénes à être uniquement lues " pour soi ».

29 Voir à ce sujet l'article très éclairant d'O. BeTTENs, "Octosyllabes, vers mesurés et effets de rythmes " précité. 


\section{Philippe Brunet et le vers d'Homère : " Non pas un calque, non pas une copie, mais un équivalent ${ }^{30}$."}

Philippe Brunet publie sa traduction de l'Iliade en 2010 aux éditions du Seuil. Contrairement à Baïf (d'après ce qu'il nous reste de son œuvre), l'auteur explique en détail sa démarche de traducteur en hexamètres dans une préface. Le propos ne vise pas tant à expliciter l'entreprise qu'à apprendre au lecteur-récitant à dire l'hexamètre, à retrouver en lui le rythme du chant aédique. "C'est bien ce travail de "rendre l'hexamètre" que j'ai tenté, plutôt que refaire ce qui avait déjà été fait pour traduire Homère ${ }^{31}$. " Cette traduction en hexamètres n'a rien à voir avec l'entreprise de Baïf, bien qu'elle puisse porter le même nom ${ }^{32}$. L'auteur explique bien que son hexamètre repose sur le retour dans chaque vers de six syllabes marquées «à la place des temps forts du grec ", l'élan dactylique étant insufflé par la première marque, systématiquement placée sur la syllabe initiale. Les marques portent sur des syllabes accentuées dans le vers, en considérant des groupes de mots réguliers ${ }^{33}$. Il semble s'agir ici d'une métrique

30 Nous citons ici ETKInd, Un art en crise, p. XV.

31 BRUnet, « Préface ", Iliade, p. 22.

32 Elle répond plutôt à une remarque faite par le traducteur lui-même dans un article portant sur de récentes traductions grecques, publié en 1991: «Dans ce contexte, les prochains traducteurs d'Homère, émules de Salel ou Jamyn, reviendront peut-être à dix ou à douze [syllabes] et recommenceront à rimer leurs Iliades. À moins qu'avides de refaire le travail perdu de Mousset, hantés par les six temps de l'hexamètre, ils ne souhaitent promouvoir une forme poétique qui n'existe pas en français. " (РH. BRUNET, Revue des Études Grecques, tome CIV (1), 1991, p. 242).

33 Cette coïncidence d'un élément métrique (le temps fort ancien, systématiquement répété) avec l'accent, dont le statut est extrêmement délicat à définir en français (métrique ? rythmique ?), de même que la nature (linguistique ? sémantique ? de hauteur ? d'intensité ? de durée ?), pose problème : s'il arrive que le lecteur puisse hésiter sur leur place, les six marques sont-elles métriques, du strict point de vue de la versification française ? Nous n'avons pas encore de réponse à cette question, mais formulerons une simple remarque : si le nombre de syllabes ne définit pas le profil métrique du vers (de façon inédite mais non moins " légitime " en poésie française), les règles prescrivant où et pourquoi une marque tombe doivent être strictes, claires et accessibles au lecteur pour sa propre expérience de scansion. Malgré les difficultés mentionnées ci-dessus pour définir l'accent, nous admettons ici qu'il existe un accent de fin de mot phonologique utilisé dans sa traduction hexamétrique par Brunet, lorsqu'il ne s'agit pas tout simplement de l'accent de mot avant ponctuation (deux accents audibles en contexte de lecture publique). Il ne faut pas oublier que cette Iliade repose sur un usage conscient de l'accentuation (explicité en préface) : elle crée donc l'attente et la recherche d'accents chez le lecteur. En lecture publique, le retour d'accents est extrêmement perceptible par l'auditoire. Si le nombre de marques n'est pas systématique (de six à quatre en général), quelque chose reste de cet hexamètre entendu, lié à la ligne mélodique des vers durant leur profération mais aussi à une diction singulièrement accentuée de la langue. À nos yeux, ces six marques se situent du côté d'un format idéal du vers, où toutes les potentialités d'accents seraient réalisées 
purement tonique. Cependant, nous le verrons plus bas, d'autres éléments sonores et rythmiques entrent en ligne de compte dans la composition et la profération d'un tel vers. Ce qui nous intéresse avant tout ici, dans cette façon de " construire " l'hexamètre, c'est précisément la nouvelle sorte de synaphie qui devrait en résulter en français : pour illustrer cela, nous reprendrons l'exemple donné dans la préface de Philippe Brunet :

Je te supplie par ta vie, tes genoux, par ton père et ta mère.

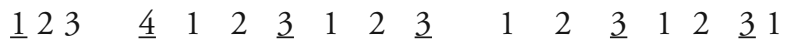

Ne laisse pas les chiens me ronger près des barques argiennes.

$$
\begin{array}{llllllllllllllll}
1 & 2 & 3 & \underline{4} & 1 & \underline{2} & 1 & 2 & \underline{3} & 1 & 2 & \underline{3} & 1 & 2 & \underline{3} & 1
\end{array}
$$

Dans ces deux hexamètres, et grâce aux marques qui sont soulignées et à cette notation numérique, l'on remarque bien que le retour des syllabes marquées ne s'effectue pas de façon totalement régulière, puisque deux syllabes marquées peuvent être séparées tantôt d'une syllabe, tantôt de deux (imitant bien, en cela, le retour des temps forts de l'hexamètre ancien, séparés, à chaque fois, par une ou deux syllabe(s), excepté lors du passage au vers suivant, ainsi la pulsation du mètre grec est-elle conservée). Cependant, ce que notent les chiffres signalés en bas du vers, c'est l'appartenance de chaque syllabe à un groupe prosodique donné, c'est-à-dire à un syntagme découpé lors de la diction ${ }^{34}$ : l'on remarque alors, en étudiant la présence des marques, que ce que

(et isolables sur le papier), tandis qu'une réalisation systématique des six accents dans les faits est évitée dans la crainte d'une perception mécanique voire monotone du vers. Quoi qu'on en dise, l'accent est lié au corps, à la subjectivation du poème (dans ce que ce terme a de plus physique) à un instant $t$ de profération. Dans l'hexamètre de Brunet en performance, toute mécanique imposant fixité est récusée, au profit de l'expression spontanée d'une voix, de l'incarnation du poème. Le rythme, conséquence d'une subjectivation du discours, dépend également de celui qui dit le vers. Le retour d'accent de l'hexamètre s'éloigne d'une métrique de papier pour rejoindre aussi la subjectivation, mais il semble bien se situer entre les deux durant la lecture : l'accent, même sans apparaître six fois de façon systématique, revient toujours à une ou deux syllabe(s) d'intervalle, et sa présence est liée à des facteurs linguistiques (puisqu'il s'appuie sur la prosodie même de la langue en y insistant) mais aussi sémantiques. Si le retour d'accent de ce vers échappe donc encore à une définition métrique claire, le "rythme " caractéristique de l'hexamètre, lui, semble se situer au cœur du passage entre vers graphique et vers proféré. Ce rythme réunit et met en jeu toutes les potentialités offertes à la fois par la métrique (qui dicte ses régularités à un certain niveau d'abstraction) et par l'interprétation du poème, sa subjectivation (qui consiste, lors de la lecture, à opérer des choix rythmico-mélodiques concrets, à incarner le verbe hexamétrique).

34 Ce groupe prosodique, constitué d'un certain nombre de syllabes et s'achevant sur un accent (qui serait réalisé en diction normale ou, pour certains cas discutables, réalisé précisément dans ce contexte "suraccentué " de l'hexamètre), coïncide souvent avec un syntagme grammatical faisant unité de sens, mais pas nécessairement, puisqu'il se place du côté de la diction et de la perception sonore, non du côté de la graphie ou de la grammaire (d'où l'appartenance du "-es» final de " barques » au groupe suivant). 
l'on pourrait appeler la première " vague " rythmique du premier vers cité se termine avant la fin du syntagme et que le début de la seconde se situe encore dans le syntagme, précisément à sa fin. Si l'on suit cette expression graphique du vers, la fin est également commencement. C'est cette superposition qui pourrait rappeler la synaphie du mètre antique : elle lie solidement les syntagmes entre eux grâce à la régularité du marquage. Pourtant, ajoutons ici une première nuance : cette synaphie serait audible pour un auditoire ancien habitué à entendre les pieds "enjamber " - si l'on nous permet l'expression - les frontières de mots. Pour un public français contemporain, il semble qu'une inversion ait tendance à s'opérer dans la perception de l'hexamètre, sans doute liée à l'accentuation descendante de la langue : de fait, la plupart des mots ou groupes de mots en français portent un accent sur la syllabe finale (sur l'avant-dernière syllabe dans les cas où un $e$ muet termine le mot ou le groupe de souffle). Ainsi, même si l'hexamètre démarre sur une syllabe marquée, le vers semble se découper ensuite selon un rythme descendant, plus anapestique (UU-) que dactylique (le poème lui-même, dans sa composition, incite à une perception anapestique : le premier vers cité montre également que le rythme U U - apparaît syntaxiquement de façon majoritaire après le second temps marqué, ces rythmes ternaires étant soulignés par la ponctuation). Plutôt que d'inversion, il faudrait parler de focalisation perceptive : même si la première syllabe est marquée, l'oreille " découpe " par syntagmes en étant par habitude sensible aux accents qui les terminent, ce qui ne veut pas dire que le public ne puisse pas percevoir ni apprécier cette inversion du rythme " initial » de la langue. Peu importe cependant qu'il s'agisse de dactyles perçus "s selon la règle " ou d'anapestes ${ }^{35}$ : il y a derrière ces vers un travail conscient du rythme, un système organisé qu'il s'agit d'analyser plus avant.

Y a-t-il systématiquement six syllabes marquées dans l'hexamètre de Philippe Brunet ? La réponse n'est pas simple : potentiellement oui. Sur le papier, d'ailleurs, lorsqu'il s'agit de scander, c'est-à-dire de chercher ces syllabes accentuées, le lecteur peut toujours noter six syllabes marquées : il en isole assez rapidement quatre, souvent cinq, qui lui semblent effectivement accentuées lorsqu'il prononce le vers à voix haute. Quand la sixième pose problème, ce qui arrive, il peut faire confiance à sa lecture personnelle du vers, mais une autre question surgit alors : la place de cette dernière marque dépend-elle de la volonté du lecteur et de l'aède, qui souhaiteraient insister sur un terme particulier dans le vers? En ce cas, toutes les marques n'auraient pas la même valeur, mais certaines témoigneraient d'une insistance "signifiante " ${ }^{36}$. Allons plus loin : dans certains hexamètres, la sixième marque pourrait bien constituer un

35 L'oreille accorde une grande importance aux découpages syntaxiques réguliers, au point d'y reconnaître une succession de cellules rythmiques équivalentes, assimilables après coup aux pieds des Anciens. Mais s'agit-il là de pieds ? Nous l'avons vu, d'un point de vue métrique, le retour d'accents mérite d'être strictement défini. Toutefois, ces marques sont assez présentes et répétées pour créer une impression de rythme régulier fort.

36 L'indécision régnant lors de la scansion pour la cinquième et la sixième marque cristallise bien le problème d'une définition de l'accent dans l'hexamètre : est-il rythmique ou 
des intermédiaires idéaux de la signifiance dont parlait Meschonnic... Pour finir, examinons deux vers : l'un où la scansion semble se dégager assez manifestement du texte, l'autre où elle pose des difficultés ${ }^{37}$. Le premier se situe au chant VI de l'Iliade, dans la supplication d'Andromaque à Hector, v. 429 :

Toi, Hector, c'est toi mon père et ma mère vaillante,

Dans ce vers, les six marques sont aisément repérables : la sémantique, la ponctuation autant que la prononciation commune des divers syntagmes incitent à accentuer six syllabes, sur lesquelles on pourrait insister en lecture publique (les accents sont présents en fin de syntagmes principaux comme sur " Hector » ou "vaillante », en fin de syntagmes secondaires, "père "/ " mère ", avec un effet d'écho sonore qui incite à accentuer le second, mais aussi sur les pronoms personnels - soulignés dans la citation - mis en apostrophe avant ponctuation forte et avec un effet d'insistance " Toi, ... c'est toi... »). Cependant, un autre exemple montre que toutes les marques ne sont pas d'emblée faciles à situer pour le lecteur : prenons le vers 453 du chant XII qui décrit Hector soulevant une roche immense pour briser la porte du mur des Achéens :

ainsi Hector la porta jusqu' aux vantaux de la porte,

Si le lecteur peut isoler d'abord les syllabes marquées de début et de fin d'hexamètre, d'emblée, les autres posent problème : bien que l'accent de fin de groupe de mots joue souvent un rôle important, il est ici remis en question par l'introduction de la préposition " jusque ", fortement liée dans ce contexte au verbe " porter ". D'où l'idée d'une recherche nécessaire des marques de l'hexamètre, car celles-ci n'y sont pas forcément apparentes si l'on se fie uniquement à une diction " commune " du vers (pour ne pas dire " naturelle »). Elles sont présentes dans l'hexamètre à l'état de potentialités : un vers d'emblée pensé comme rythmé (hexarythmé pourrait-on dire) laissera apparaître ses syllabes accentuées plus facilement : ici sur les fins de syntagmes (" porta ", "vantaux "), mais aussi sur la finale du nom du héros. La sixième marque posera problème, puisqu'elle ne saurait être placée ailleurs que sur " aux ", difficilement accentuable en français sans quelque artificialité audible. Là encore, il n'est pas obligatoire de la réaliser. La seule raison qui pourrait pousser le lecteur à s'appesantir sur cette syllabe serait de vouloir mimer, par un allongement vocalique répété dans le vers, l'effort d'Hector soulevant cette pierre, en reprenant notamment l'amorce spondaïque de l'hexamètre d'Homère. En effet, bien qu'un tel allongement puisse être discuté, au vu du sens même de la comparaison homérique (Hector est comparé à un berger qui

métrique ? Si la fixation des accents dépend de facteurs différents dans le même vers (linguistiques, axiologiques), la thèse rythmique semble l'emporter.

37 Un troisième exemple cristallise le problème de la double accentuation d'un mot placé en début d'hexamètre pour obtenir six marques : " boucliers au bel arrondi et rondaches légères " (XII, v. 426). S'il est " placé " en scansion, cet accent initial pourra être atténué ou abandonné à la diction, évitant l'irruption d'une étrangeté acoustique. 
soulève d'une seule main - c'est-à-dire avec facilité - la toison d'un bélier fraîchement tondu), il ne faut pas oublier que la lourdeur même de cette roche était soulignée des vers 446 à 450 dans une description qui se clôturait sur l'allègement de cette roche par Zeus venant en aide au héros. Dans le texte homérique, au vers 453, la lourdeur naturelle de cette pierre est donc à la fois récusée par la comparaison (et le secours surnaturel du dieu) et présente dans l'esprit de l'auditoire. C'est là que se loge, sans doute, la signifiance, dans l'amorce doublement spondaïque du vers chez Homère (qui renvoie à cette lourdeur effective de la roche rendue temporairement légère), et, potentiellement, dans l'allongement possible de ces voyelles dans l'hexamètre français. L'équivalence permet à la signifiance de ne pas se perdre, à l'agir du poème d'être transmis, forme et sens agissant de concert. Ce qui est certain, c'est que dans le contexte de performance, les six marques ne sont pas systématiquement réalisées : la première surtout, qui est " censée " donner son élan dactylique à l'hexamètre, n'est absolument pas obligatoire, surtout s'il l'on a affaire à un mot inaccentué et "inaccentuable ${ }^{38}$ » en position initiale. Le rythme des cinq autres marques se dessine rapidement, puisqu'elles sont entrecoupées d'une ou deux syllabe(s). Le fait est qu'il ne s'agit pas ici d'un patron métrique rigide qu'il s'agirait de réaliser, tel que nous l'avons exploré chez Baïf. La fonction de cet hexamètre est claire : il doit être dit, et même transmis. Dans ce contexte de transmission à un public, c'est l'aède qui a la primeur et décide de ce que doit faire le vers, si nous voulons emprunter l'idée à Meschonnic. Il ne s'agit donc en rien d'un système rigide ou d'un calque. L'élan rythmique prime, avec son retour de temps marqués, réalisant plus ou moins complètement un schème métrique initial que l'aède garde à l'esprit. Le schème reste un ensemble de potentialités toutes plus ou moins exploitées selon l'hexamètre proféré.

Cette nuance nous permet de passer à ce qui différencie précisément l'hexamètre baïfien de l'hexamètre de Philippe Brunet : le premier compte avant tout sur la compréhension par le lecteur d'un patron rigide, rendu en partie lisible par la graphie particulière des Étrénes et certes réalisé à la lecture à haute voix, mais dont la poétique de traduction repose sur un procédé de calque direct du mètre ancien. L'hexamètre est restitué comme il a été reçu, en tant qu'objet "pur ", presque inaltéré. Celui que l'on trouve dans cette traduction récente de l'Iliade réalise les souhaits d'E. Etkind dans son ouvrage de 1982 : il s'agit là non d'une copie mais de la création d'un équivalent, et même d'une recréation. Le traducteur a retenu de l'hexamètre ce qu'il pouvait en faire passer dans sa langue, non sans la contraindre un tant soit peu de manière assumée (rappelons-nous cette exhortation dans la préface de la Théogonie : "Faut-il aller dans le sens de la langue ou aller contre elle ? La question aurait fait rire Thalie. Il y a

38 Avec toutes les précautions qu'il faut mettre dans le passage d'un adjectif à l'autre, voir à ce sujet B. DE CORNULIER, "La place de l'accent, ou l'accent à sa place", dans Le vers français. Histoire, théorie, esthétique, M. Murat (dir.), Paris, Honoré Champion, 2000, p. 66-67. 
certainement plus d'une réponse possible. Si la nature résiste, tant mieux : que la lutte soit âpre. Le résultat ne dépend pas seulement de la théorie ${ }^{39}$.")

De ce point de vue, il est tout à fait intéressant d'étudier la façon dont Brunet utilise les propriétés inhérentes au français pour créer un hexamètre dans cette langue. Même si tous les vers ne débutent pas sur une syllabe marquée, le traducteur donne fréquemment à son hexamètre l'élan du dactyle en introduisant un accent initial qui se profile en lecture intérieure et dont la réalisation sera surtout audible en performance. À cette fin, plusieurs procédés sont employés : l'introduction d'interjections, de noms propres et communs monosyllabiques mis en apostrophe (tel "Zeus", ou encore l'insulte "Chien!" lancée par Achille à Hector échappant à ses traits, v. 449, XX) dans les deux cas suivis d'une ponctuation forte, d'impératifs monosyllabiques suivis de points d'exclamation, ou encore l'insertion - à fonction emphatique en contexte de pronoms personnels monosyllabiques suivis d'une virgule. Notons que, ne serait-ce que d'un point de vue sémantique, ce type d'initiales peut toujours faire l'objet d'une accentuation d'insistance. Outre ces monosyllabes, certains dissyllabes à finales en « e " muet, prononcées plus faiblement dans l'hexamètre et par là presque assimilables à des monosyllabes, peuvent porter une marque initiale lorsqu'ils sont placés en apostrophe suivie d'une ponctuation forte ${ }^{40}$ (Achille qualifie ainsi Agamemnon de "Monstre de rapacité, [...] » v. 149, I). L'utilisation de mots composés, dont l'accentuation reste problématique dans la langue, permet aussi d'introduire un accent initial sans choquer l'oreille : «Face-de-chien ! », v. 160, I (Achille s'adressant encore au roi de Mycènes). Grâce au "rebond » bref permis par le " e " muet, prononcé plus faiblement mais aussi plus rapidement dans le vers, plusieurs attaques sonnent proprement dactyliques, si l'on veut bien assumer que dans ces cas précis l'accent donne à la syllabe marquée un relief, une intensité voire une durée différents de ceux des autres syllabes du vers ${ }^{41}$. En outre, si l'on s'attache également à ce qui suit l'attaque du vers, l'on s'aperçoit que Philippe Brunet a recours à d'autres techniques pour introduire les marques de l'hexamètre. Entre autres, nous citerons ici encore l'emploi de mots composés qui permet l'introduction d'une multiplicité d'accents (on pense à Calchas, dans le souvenir d'Ulysse, parlant d'un signe de Zeus : " tard-venu, tard-accompli », II, v. 325), mais aussi la traduction des diverses épithètes homériques : tantôt rendues par des mots composés

39 Hésiode, La Théogonie, Les Travaux et les Jours et autres poèmes, trad. PH. BRUNET, LGF, 1999, "Préface ", p. 12-13.

40 La ponctuation " dénonce " à maintes reprises la présence de certaines marques, aidant ainsi le lecteur désireux de faire résonner l'hexamètre pour lui-même ou bien pour un auditoire.

41 L'allongement de la voyelle prononcée sous l'accent devrait être phonologiquement repérable après enregistrement et analyse d'une profération du vers, elle est du moins admise comme possible dans la préface de BRUNET, p. 23-24 ; là également se trouve, dans le rythme restitué, la trace de la prosodie ancienne (non uniquement les six temps forts, mais aussi, mutatis mutandis, une alternance de durées). 
(pour Poséidon par exemple "tremble-terre "), tantôt par des syntagmes composés d'un nom et de son complément (citons un vers assez remarquable de ce point de vue, le vers $43 \mathrm{du}$ chant XIII, où les épithètes homériques, traduites suivant divers jeux d'échos sonores, scandent littéralement le phrasé : "Mais Poséidon, le Socle du sol, l'Ébranleur de la terre "), elles permettent presque toujours d'introduire plusieurs accents, répondant ainsi aux principes d'élaboration concertée de l'hexamètre définis dans la préface. Ces recherches dans la traduction des épithètes, à la fois sémantiques, rythmiques et sonores, révèlent qu'il s'agit là d'une traduction façonnée à voix haute, d'un texte avant tout proféré pour pouvoir être choisi ${ }^{42}$.

\section{Ce que l'hexamètre est devenu}

Plutôt que de les concevoir comme une série d'expériences dérangeantes pour les chercheurs en métrique et en littérature, il est possible de considérer les tentatives de restitution des mètres antiques en français comme autant d'occasions de réinterroger un ensemble de notions clefs en métrique, en poétique ou encore en traductologie. Nous pensons par exemple à la notion de "génie de la langue française " ou encore à la définition des multiples rapports que le rythme peut entretenir avec le sens. Ces expériences constituent de remarquables terrains d'exploration des potentialités de la langue française, d'une part du point de vue rythmique et sonore, d'autre part - et tout à la fois ! dirait Meschonnic - du point de vue du sens. L'hexamètre choisi pour traduire l'épopée ancienne ne se veut pas une solution de traduction plus "naturelle " que la métrique syllabique. Car qu'est-ce qui, en matière de métrique, est "naturel " ? Ce que nous apprennent Jean-Antoine de Baïf et Philippe Brunet, ce n'est pas à scander, un crayon à la main, un texte qui nous semblerait alors obscur, mais bien à ressentir la poésie et le sens, dans le mouvement même du rythme initial, ou plutôt dans un élan d'aspiration enthousiaste vers ce rythme. Des milliers de vers épiques, capables grâce à l'ambition singulière de plusieurs expérimentateurs, de créer cette "poétique du continu ", idéal de Meschonnic. Avant toute chose, autant de milliers d'hexamètres qui permettent à la voix du poète originel d'être redoublée, à travers l'acte de recréation

42 Notons cependant que ce texte n'a été fixé que pour les besoins de l'édition, le traducteur lui-même se méfiant de toute fixité en matière de traduction du poème homérique, voir préface, p. 28-29. Ajoutons que ce travail sur les sonorités est rendu possible aussi par l'emploi d'un lexique poétique extrêmement riche en traduction, rappelant l'infinie richesse lexicale d'Homère. Nous pensons par exemple à la traduction de cette comparaison des vieux conseillers de Priam, perchés sur le rempart de Troie, à des cigales chantant dans un arbre : v.150-153, III : " Mais pour l'éloquence, / ils étaient agiles! Pareils aux cigales dans l'arbre, / qui font jaillir en forêt leur stridulation liliale. " Le dernier groupe nominal, distingué par sa rareté d'usage en français, rend ici de façon délicatement sonore l'impression poétique du chant imaginé par Homère. 
que constitue la traduction, par celle du poète-traducteur. Poussés par un même élan, Baiff et Brunet travaillent en somme différemment : le premier prévoit six positions lourdes obligatoires dans son vers, disposant les syllabes selon une prosodie du français bien réglée, de façon à faire entrer celles-ci dans un moule prédéterminé (et, dans l'idéal, quantitatif) : le patron métrique classique. Le second prévoit de marquer six syllabes dans un processus de restitution de l'impression rythmique laissée par l'hexamètre grec et communicable en français. Les six syllabes lourdes de Baif comme les six syllabes marquées de l'hexamètre de Brunet ne constituent pas à elles seules le caractère poétique du texte, mais elles permettent de lui donner ce rythme particulier qui, sans être exactement celui d'Hésiode ou d'Homère, crée une nouvelle parole-poème, parfois à la frontière entre parole et chant. Parole scandée. Un mouvement s'inscrit dans le vers et vise à gagner le corps de celui qui lit ou de celui qui écoute. Ce mouvement est aussi celui qui va de "rendre l'hexamètre " à « recevoir l'hexamètre ». Pour le recevoir, il faut d'emblée accepter cette première sensation, rare et singulière, la sensation de percevoir quelque chose d'étranger dans sa propre langue. Cet étrange dépassé, ou plutôt considéré comme un acquis nouveau, la langue que l'on parle se révèle alors bien plus riche, plus vivante qu'il n'y paraît. Une fois encore, il y a poésie.

Juliette LORMIER

ALITHILA (Université de Lille 3)

Rue du Barreau

PB 60149

59653 Villeneuve-d'Asq Cedex

juliette.lormier@gmail.com

\section{Bibliographie succincte}

M. Augé-Chiquet, La Vie, les idées et l'œuvre de Jean-Antoine de Baïf, Hachette-E. Privat, Paris-Toulouse, 1909.

J.-A. de BAïr, Étrénes de poézie fransoeze an vers mezurés. Les Besognes \& Jours d'Hésiode \& quelques Odes, Paris, 1574 ; repris dans Étrénes de poézie fransoeze an vers mezurés; Psautier en vers mesurés, Genève, Slatkine (réimpr.), 1972.

O. BetTens, "Octosyllabes, vers mesurés et effets de rythmes ", dans L'expérience du vers en France à la Renaissance, J.-C. Monferran (dir.), Cahiers Saulnier, $\mathrm{n}^{\circ} 30$, Paris, Presses Universitaires Sorbonne, 2013, p. 185-213.

B. de Cornulier, "La place de l'accent, ou l'accent à sa place ", dans Le vers français. Histoire, théorie, esthétique, M. Murat (dir.), Paris, Honoré Champion, 2000, p. 66-67.

E. ETKInd, Un art en crise. Pour une poétique de la traduction poétique, Paris, L'Âge d'Homme, 1982. 
Hésiode, La Théogonie, Les Travaux et les Jours et autres poèmes, traduction de $\mathrm{Ph}$. BRunet, commentaires de M.-Ch. LeClerC, Paris, Librairie Générale Française, coll. « Le Livre de poche ", 1999.

Homère, L'Iliade, traduit du grec par Ph. BRUNET ; préface, notes et répertoire établis par le traducteur, Paris, Seuil, 2010.

J. IRIGOIN, "Césure et diction du vers. Quelques réalités linguistiques à ne pas oublier ", dans Autour de la césure. Actes du colloque Damon des 3-4 novembre 2000, M. Steinrück et A. Lukinovich (dir.), Berne-Berlin-New York, F. Spaltenstein, O. Bianchi (éd.), Peter Lang, 2004, p. 1-10.

H. Meschonnic, Poétique du traduire, Lagrasse, Verdier, 1999.

Y.-Ch. Morin, "La graphie de Jean-Antoine de Baïf : au service du mètre ", Nouvelle Revue du seizième siècle, 17 (1), 1999, p. 85-106.

Y.-Ch. Morin, «L'hexamètre "hérö̈que" d'Antoine de Baï », dans Métriques du Moyen Âge et de la Renaissance, D. Billy (dir.), Paris-Montréal, L’Harmattan, 1999, p. 163-184.

Y.-Ch. Morin, " La prononciation et la prosodie du français du XVI ${ }^{e}$ siècle selon le témoignage de Jean-Antoine de Baï ", Langue française, $n^{\circ} 126,2000$, p. 9-28.

G. Peureux, La fabrique du vers, coll. "Poétique », Paris, Seuil, 2009. 